\title{
Cavitation Flow of Oil and Water Through the Nozzle
}

\author{
Marian Bojko ${ }^{1}$, Milada Kozubková, ${ }^{1, *}$, and Jana Jablonská ${ }^{1}$ \\ ${ }^{1}$ VSB - Technical University of Ostrava, Faculty of Mechanical Engineering, Department of \\ Hydromechanics and Hydraulic Equipment, 17. listopadu 2172/15, 70800 Ostrava-Poruba, Czech \\ Republic
}

\begin{abstract}
The hydraulic equipment and elements are designed so that the flow is not significantly affected by the content of gases in the fluid. In the case of cavitation, there is a change in the volumetric amount of gas, which in water is due to the air and water vapour present, and in the case of oils, especially the air content. This phenomenon causes a significant change in the loss coefficient of the element. The problem of cavitation is solved in the literature for water flow, for other hydraulic fluids (e.g. hydraulic oils operated at different temperatures) the problem is still not solved to a sufficient extent. The article deals with the issue of cavitation in systems in which different types of liquids are used. In the introduction, the physical properties of the used liquids are evaluated, because they significantly influence the origin and development of cavitation. Subsequently, an experimental device with a transparent nozzle is described, on which the measurement. The dependence of the loss coefficient and the cavitation number on the Reynolds number is evaluated. Cavitation is evaluated by a high-speed camera, where it is possible to monitor the behaviour of the cavitation cloud.
\end{abstract}

\section{Introduction to the issue}

Cavitation and cavitation wear are constantly recurring problem in hydraulic systems. Cavitation wear devalues hydraulic components in the hydraulic circuit, especially pumps. The study of cavitation in the flow of water is very complicated, because the cavitation cloud consists of vapour and gas (air). Examination of cavitation in water flow is very complicated, because vapour and gas (air) cavitation arises during the cavitation of water. The flowing oil does not evaporate and only gaseous (air) cavitation occurs, which is given due to the saturated vapour pressure $[4,6]$. Both types of cavitation similarly affect the magnitude of local resistance.

The comparative experiment is based on the flow of hydraulic fluids (oil and water) in a convergent divergent nozzle (CD nozzle).

\footnotetext{
* Corresponding author: milada.kozubkova@vsb.cz
} 


\section{Physical properties}

At higher temperatures, the liquid (oil, water) is more susceptible to cavitation. This is mainly due to the physical properties of the liquid and the saturated vapour pressure, which can be significantly temperature dependent.

Table 1 compares physical properties of liquids that affect the formation and size of cavitation. These are mainly density, modulus of volume elasticity of the liquid, surface tension and viscosity. Most of the properties are mainly temperature dependent, so it is necessary to know the heat balance in hydraulic circuits [8].

Table 1. Table of physical properties affecting cavitation [1].

\begin{tabular}{|l|c|c|c|c|}
\hline & \multicolumn{4}{|c|}{ liquid } \\
\hline physical properties at $20^{\circ} \mathrm{C}$ & water & mineral oil & $\begin{array}{c}\text { polyglycol - } \\
\text { water solution }\end{array}$ & $\begin{array}{c}\text { synthetic } \\
\text { liquid }\end{array}$ \\
\hline density $\left[\mathrm{kg} \cdot \mathrm{m}^{-3}\right]$ & 1000 & 870 to 900 & 1010 to 1090 & 1100 to 1300 \\
\hline $\begin{array}{l}\text { modulus of volume } \\
\text { elasticity of fluid }[\mathrm{Pa}]\end{array}$ & $2.2 \cdot 10^{9}$ & $1.6 \cdot 10^{9}$ & $(3$ to 3.5$) \cdot 10^{9}$ & $(2.3$ to 3$) \cdot 10^{9}$ \\
\hline surface tension $\left[\mathrm{N} \cdot \mathrm{m}^{-1}\right]$ & 0.073 & 0.035 & $(0.03$ to 0.04$)$ & $(0.025$ to 0.045$)$ \\
\hline the solubility of air $[1]$ & 0.02 & 0.08 to 0.1 & 0.01 to 0.02 & 0.08 to 0.09 \\
\hline kinematic viscosity $\left[\mathrm{m}^{2} \cdot \mathrm{s}^{-1}\right]$ & $1 \cdot 10^{-6}$ & $1.02 \cdot 10^{-4}$ & $0.89 \cdot 10^{-4}$ & $1.48 \cdot 10^{-4}$ \\
\hline
\end{tabular}

Publication 2 states that the concentration of non-condensable gas in water at $20{ }^{\circ} \mathrm{C}$ is $2-$ $3 \%$ (approximately $15 \mathrm{ppm}$ ) and the concentration in the oil is above $8 \%$ (approximately $40 \mathrm{ppm}$ ). Thus, the concentration of non-condensable gas in water and in viscous oils with a viscosity of $24 \mathrm{cS}=2,4 \cdot 10^{-5} \mathrm{~m}^{2} . \mathrm{s}^{-1}$ is assigned to $15 \mathrm{ppm}$; the concentration in oils with a viscosity higher than $24 c S$ is $40 \mathrm{ppm}$ [2]. Both publications [1,2] agree in the amount of air contained in water and oil.

Figure 1 evaluates the density and dynamic viscosity of mineral oil (VG46) and water depending on the temperature, see lit [5]. It is clear that the most significant changes are observed in the oil viscosity. These dependencies are used for further evaluation.

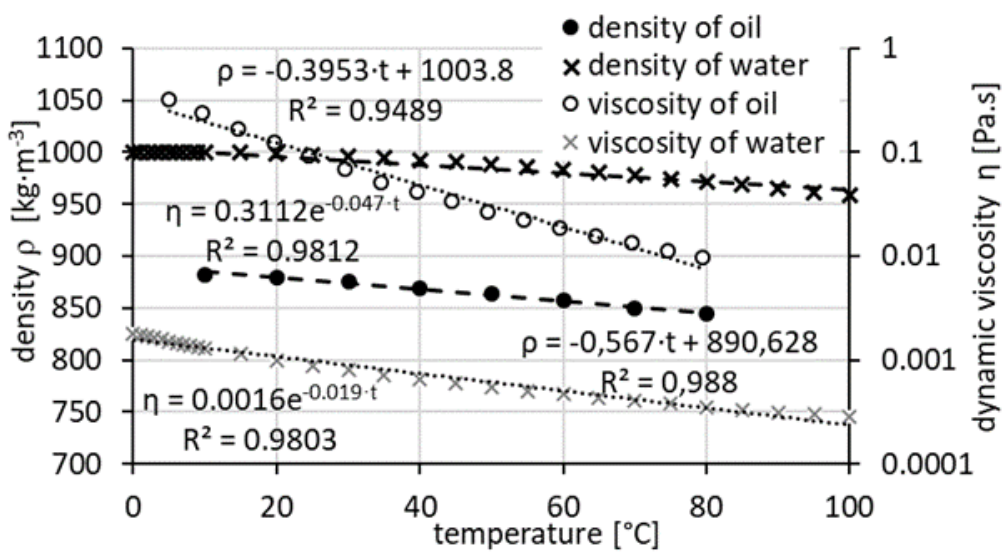

Fig. 1. Dependence of density and dynamic viscosity vs. temperature.

The saturated vapour pressure for water increases with increasing temperature. Water at $25^{\circ} \mathrm{C}$ was used in the experiment, which corresponds to a saturated vapour pressure $p_{w}=3166 \mathrm{~Pa}$. However, cavitation of the oil at this temperature $\left(25^{\circ} \mathrm{C}\right)$ was not observed and did not manifest itself significantly until the temperature of $50^{\circ} \mathrm{C}$, which corresponds to the saturated vapour pressure for the oil $p_{w}=0.0047 \mathrm{~Pa}$. The saturated vapour pressure for water at $50{ }^{\circ} \mathrm{C}$ is $p_{w}=12335 \mathrm{~Pa}$. Remarkable is the large difference between the values for 
water and mineral oil, or for synthetic liquids $\left(p_{w}=2 \mathrm{~Pa}\right.$ at $50{ }^{\circ} \mathrm{C}$ ). Emulsions (polyglycolwater solution $p_{w}=8000 \mathrm{~Pa}$ at $50{ }^{\circ} \mathrm{C}$ ) contain $35 \%$ to $50 \%$ water, therefore their saturated vapour pressure is close to the saturated vapour pressure of water. All values are given at atmospheric pressure [1].

\section{Experiments}

A hydraulic circuit was designed and implemented at the workplace to investigate the cavitation properties during the flow of liquids through the nozzle. From the tank (1) the liquid is pumped by the hydrogenerator (2) into the circuit, where a ball valve (3) for flow control and a flow meter (4) is subsequently placed. Furthermore, the measured element (5) is included in the circuit, i.e. a transparent circular convergent divergent nozzle of a given shape with an inlet diameter $\emptyset 20 \mathrm{~mm}$ and a diameter at the narrowest section $\emptyset 6 \mathrm{~mm}$. Pressure sensors (6) are located in front of and behind the nozzle, data are recorded and evaluated in the device (7). The pipe is led below the level in the tank (1), where the thermometer (8) is located.

In the case of the oil circuit, a regulating hydrogenerator with a tilting plate is used, the CD nozzle is placed vertically, there is a possibility of heating the liquid in the circuit. In the case of a water circuit, the pump is regulated by a frequency converter, the CD nozzle is placed horizontally.

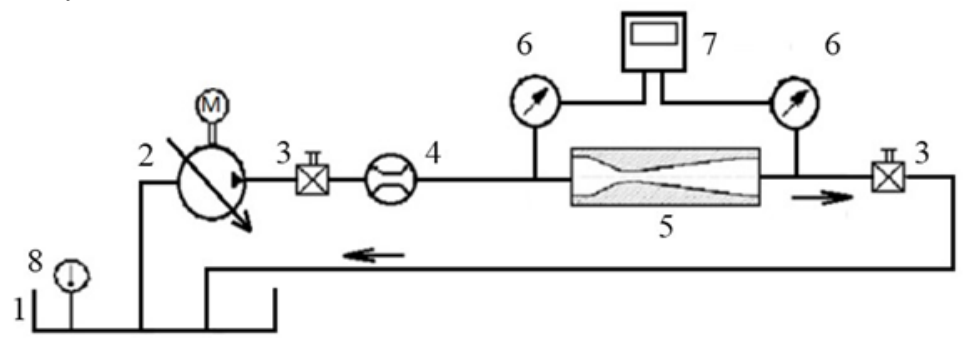

Fig. 2. Measuring line diagram - 1 tank, 2 hydrogenerator / pump, 3 ball valve, 4 flow meter, 5 convergent divergent nozzle, 6 pressure sensors, 7 evaluation and recording equipment, 8 thermometer.

\subsection{Evaluation of measurement of hydraulic parameters}

The initial measurements of hydraulic parameters were performed on the water pipe and on the oil pipe for different fluid temperatures. On the oil pipe, the values of the measured volume flow rate are in the range from $1.7 \cdot 10^{-4} \mathrm{~m}^{3} \cdot \mathrm{s}^{-1}$ to $5.86 \cdot 10^{-4} \mathrm{~m}^{3} \cdot \mathrm{s}^{-1}$, On the water pipe, the values are from $1.72 \cdot 10^{-4} \mathrm{~m}^{3} \cdot \mathrm{s}^{-1}$ to $7.23 \cdot 10^{-4} \mathrm{~m}^{3} \cdot \mathrm{s}^{-1}$. Figure 3 presents the dependence of the pressure gradient on the volume flow rate. With increasing volume flow rate, the pressure drops for water and oil are different, but the values are increasing. The viscosity of the oil decreases with increasing temperature and thus the pressure loss decreases. The dashed line indicates the cavitation limit that was evident from the measurement. 


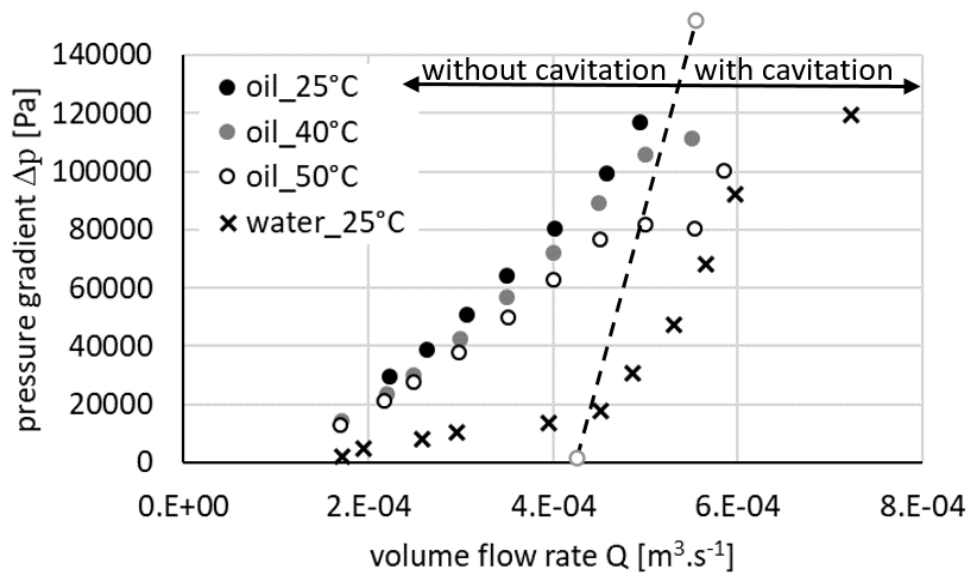

Fig. 3. Dependence of pressure gradient vs. volume flow rate.

Measurements for water were performed at $25{ }^{\circ} \mathrm{C}$. The measurements for the oil were performed at the same temperature, but cavitation was not achieved, so the oil was heated and the dependence of the pressure drop on the flow at the temperature $40{ }^{\circ} \mathrm{C}$ and $50{ }^{\circ} \mathrm{C}$ is also evaluated. The cavitation has already been achieved at these temperatures.

The dependence of the dimensionless quantities is evaluated in Figure 4, i.e. the dependence of cavitation number on the Reynolds number defined for the narrowest cross section of the CD nozzle, where the Reynolds number is defined as [1, 4]

$$
\operatorname{Re}=\frac{v \cdot d}{v}=\frac{v_{\max } \cdot d_{\min }}{v}
$$

and the cavitation number is defined $[1,4]$

$$
\sigma=\frac{2 \cdot\left(p_{\text {in }}-p_{w}\right)}{\rho \cdot v_{\max }^{2}}
$$

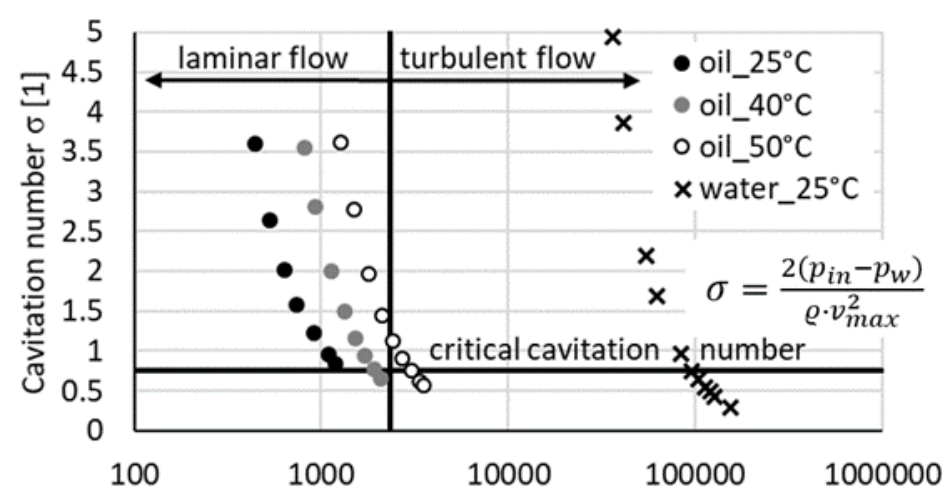

Reynolds number in the narrowest cross section Re [1]

Fig. 4. Dependence of cavitation number vs. Reynolds number in the narrowest cross section of CD nozzle.

The authors assume a critical Reynolds number 2320 for laminar resp. turbulent flow through circular cross section. It can be seen from the graph that the oil flow is moving primarily in the laminar region, the Reynolds number at the inlet to the nozzle is in the range from 130 to 1055 . Reynolds number in the narrowest cross section the CD nozzle is slightly 
larger. The Reynolds number in the narrowest cross section of the CD nozzle at water flow is in the range from 36497 to 153525 and at the inlet to the nozzle it is in the range from 10949 to 46058 . The critical value of the cavitation number was determined from the experiment as 0.747 and is in agreement with the literature [3].

The loss factor $\xi$ and subsequently the resistance coefficient $R$ can be determined from the basic theory of fluid mechanics for turbulent flow (Bernoulli's equation) [1]

$$
\Delta p=\xi \cdot \rho \cdot \frac{v^{2}}{2}=\xi \cdot \rho \cdot \frac{1}{2 \cdot S^{2}} Q^{2}=\xi \cdot \rho \cdot \frac{16}{2 \cdot \pi^{2} \cdot d^{4}} Q^{2} \Rightarrow \Delta p=R Q^{2}
$$

then resistance coefficient $R$ can be determined as follows

$$
\Delta p=R \cdot Q^{2} \Rightarrow R=\frac{\Delta p}{Q^{2}}
$$

By comparing equations (3) and (4) it is possible to obtain the relationship between the resistance coefficient $R$ and the loss coefficient $\xi$

$$
\mathrm{R}=\frac{\xi \cdot \rho}{2 \cdot S^{2}} \Rightarrow \xi=\frac{2 \cdot \mathrm{R} \cdot S^{2}}{\rho}
$$

It can be seen from Figure 5 that the loss coefficient is significantly higher with laminar oil flow than with turbulent water flow. The loss coefficient is given by the physical properties of the flowing liquid. It can be seen from equation (3) that it indirectly depends on the density of the flowing liquid and the square of the velocity. The loss coefficient for oil and especially for water is significantly affected by cavitation and does not correspond to the current trend given by the theory of fluid mechanics, therefore to determine the loss coefficient it is necessary to consider only experimental values measured without cavitation.

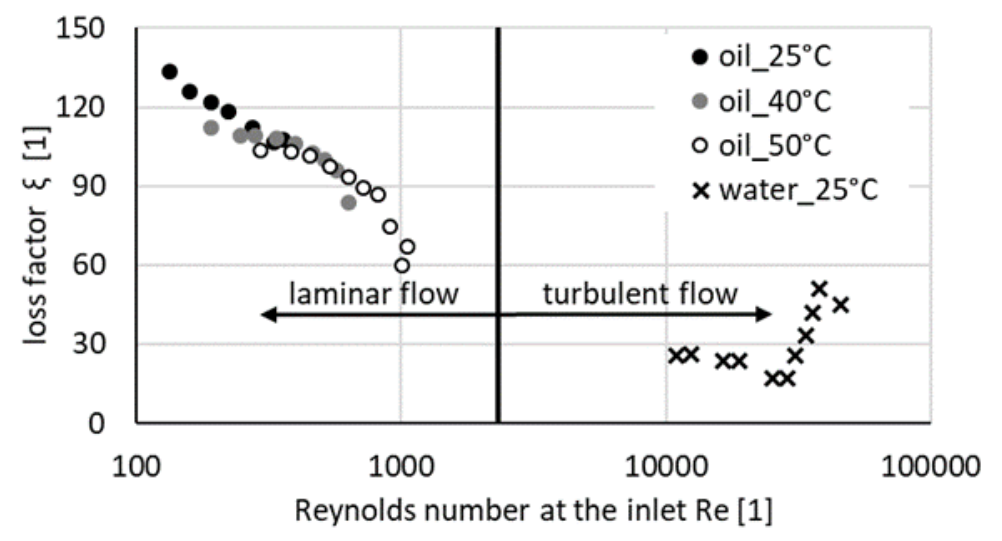

Fig. 5. Dependence of loss factor vs. Reynolds number at the inlet.

The dependence of pressure drop on the square of the volume flow rate is plotted at Figure 6, from this it is possible to determine the resistance coefficient $R$ and subsequently the loss coefficient $\xi$ using the least squares statistical method. It is clear from the derivation that the lines must pass through the beginning of the coordinate system. 


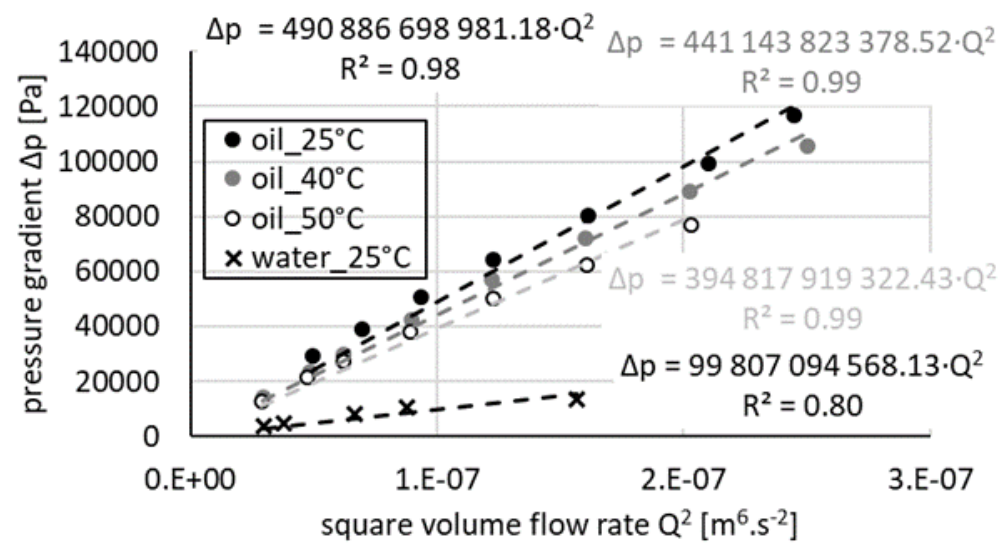

Fig. 6. Dependence of pressure gradient vs. square volume flow rate.

From the equations in Figure 6, the loss coefficients are calculated using equation (3) and subsequently evaluated in Figure 7. It can be seen that for laminar oil flow the loss factor is considerably higher than for turbulent water flow. The loss coefficient evaluated in this way can also be used in the classical design of hydraulic equipment, where are not expected to ordinarily cavitation mode.

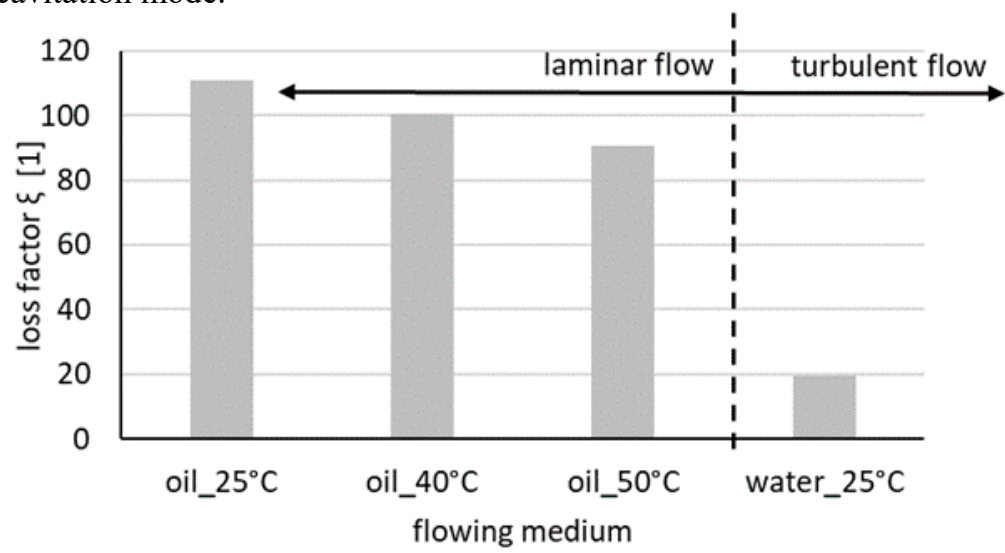

Fig. 7. Dependence of loss factor vs. flowing medium.

\subsection{Evaluation of the cavitation cloud}

The experimental device was designed to be able to watch the size of the cavitation cloud depending on the type of flowing medium and its temperature and the size of the cavitation bubbles. Significant dynamic behaviour of the cavitation cloud can also be observed during cavitation.

Figure 8 shows the size of the cavitation area for oil. Variant a) is $40^{\circ} \mathrm{C}$ for oil and volume flow rate $Q=5.5 \cdot 10^{-4} \mathrm{~m}^{3} \cdot \mathrm{s}^{-1}(\sigma=0.655)$ variant b) $50{ }^{\circ} \mathrm{C}, Q=5 \cdot 10^{-4} \mathrm{~m}^{3} \cdot \mathrm{s}^{-1}(\sigma=0.747)$, c) $50{ }^{\circ} \mathrm{C}, Q=5.54 \cdot 10^{-4} \mathrm{~m}^{3} \cdot \mathrm{s}^{-1}(\sigma=0.621)$, d) $50^{\circ} \mathrm{C}, Q=5.86 \cdot 10^{-4} \mathrm{~m}^{3} \cdot \mathrm{s}^{-1}(\sigma=0.563)$. Variant a), b) is an initial cavitation, which is more pronounced at a greater distance behind the constriction. Variant c) and d) is already fully developed air cavitation [7]. 


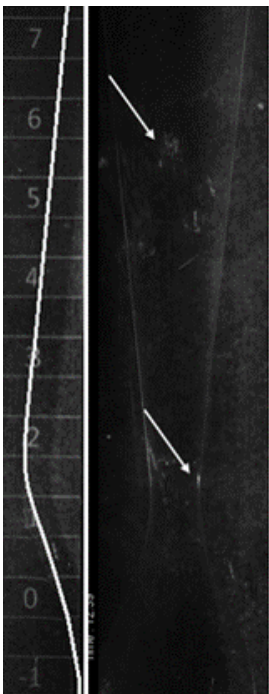

a)

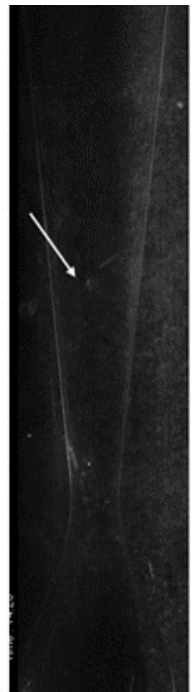

b)

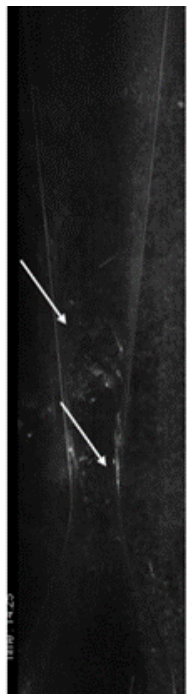

c)

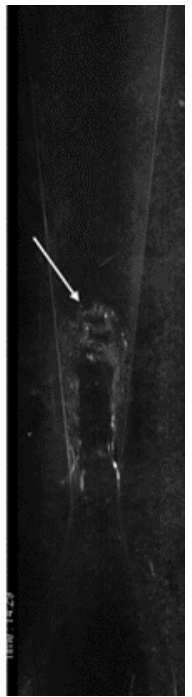

d)

Fig. 8. Cavitation area size for oil (scale is in centimetres) a) $40{ }^{\circ} \mathrm{C}, \sigma=0.655 \mathrm{~b}$ ) $50{ }^{\circ} \mathrm{C}, \sigma=0.747$ c) $50^{\circ} \mathrm{C}, \sigma=0.621$ d) $50^{\circ} \mathrm{C}, \sigma=0.563$.

Figure 9 shows the size of the cavitation area for water. Variant a) $Q=4.51 \cdot 10^{-4} \mathrm{~m}^{3} \cdot \mathrm{s}^{-1}$ $(\sigma=0.747)$ represents the initial cavitation, which manifests itself on the nozzle walls in the constriction. Variants b) $Q=4.87 \cdot 10^{-4} \mathrm{~m}^{3} \cdot \mathrm{s}^{-1}(\sigma=0.651)$, c) $Q=5.531 \cdot 10^{-4} \mathrm{~m}^{3} \cdot \mathrm{s}^{-1}(\sigma=0.552)$, d) $Q=5.566 \cdot 10^{-4} \mathrm{~m}^{3} \cdot \mathrm{s}^{-1}(\sigma=0.486)$, represent a transition to fully developed cavitation, where the nozzle area is not completely filled with gas, but the cavitation is clearly visible. Variant e) $\left.Q=5.98 \cdot 10^{-4} \mathrm{~m}^{3} \cdot \mathrm{s}^{-1}(\sigma=0.433) \mathrm{f}\right) Q=7.23 \cdot 10^{-4} \mathrm{~m}^{3} \cdot \mathrm{s}^{-1}(\sigma=0.295)$ represents a fully developed cavitation, where a cloud of steam and air fills the entire cross section of the nozzle.

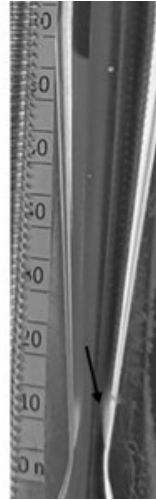

a)

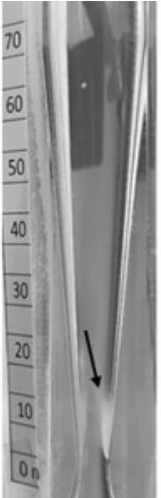

b)

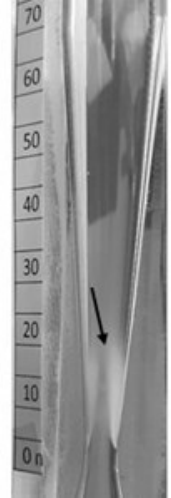

c)

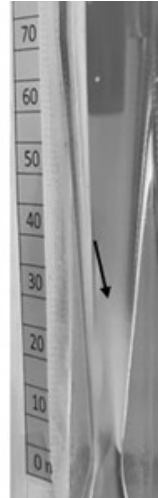

d)

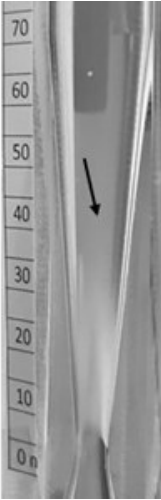

e)

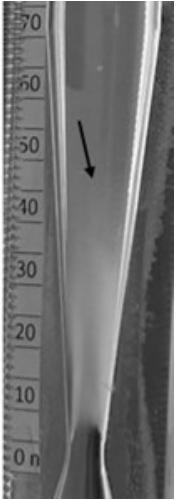

f)

Fig. 9. Cavitation area size for water (scale is in millimetres) a) $\sigma=0.747 \mathrm{~b}$ ) $\sigma=0.651 \mathrm{c}$ ) $\sigma=0.552$ d) $\sigma=0.486 \mathrm{e}) \sigma=0.433 \mathrm{f}) \sigma=0.295$.

If we compare the size of the developed cavitation area for the same flows (Figure 10), i.e. for oil $50^{\circ} \mathrm{C}$ variant $\sigma=0.621\left(Q=5.54 \cdot 10^{-4} \mathrm{~m}^{3} \cdot \mathrm{s}^{-1}\right)$ and for water variant $\sigma=0.486$ $\left(Q=5.566 \cdot 10^{-4} \mathrm{~m}^{3} \cdot \mathrm{s}^{-1}\right)$, it is evident that the cavitation area is slightly larger in water.

If we compare the size of the cavitation area for approximately the same cavitation numbers, i.e. for oil variant d) $Q=5.86 \cdot 10^{-4} \mathrm{~m}^{3} \cdot \mathrm{s}^{-1}(\sigma=0.563)$ and for water variant 
c) $Q=5.531 \cdot 10^{-4} \mathrm{~m}^{3} \cdot \mathrm{s}^{-1}(\sigma=0.552)$, the cavitation region of the oil is clearly larger. From this it is clear that the size of the cavitation area again depends on the physical properties of the liquid. It was confirmed that cavitation on a given geometry originated at the same cavitation number, but subsequently the size of the cavitation cloud is different.

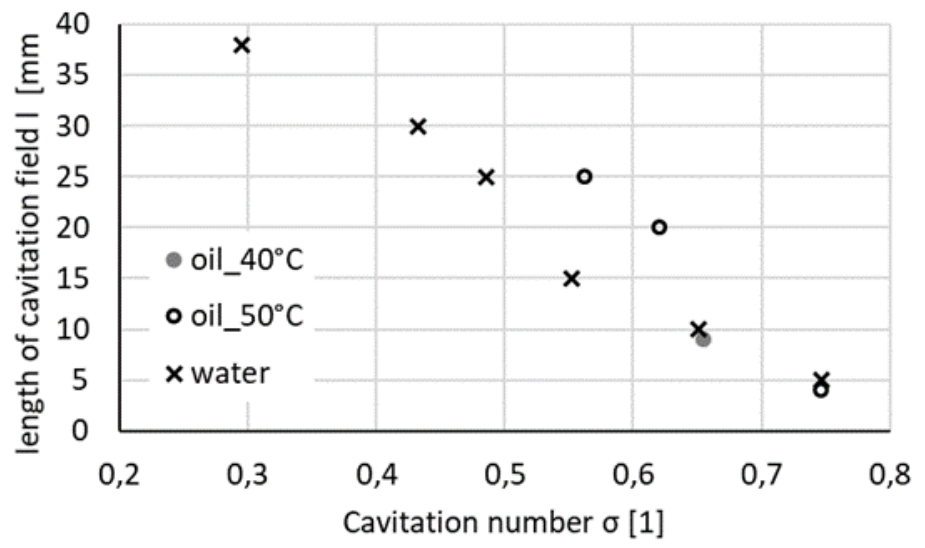

Fig. 10. Dependence of length of cavitation field vs. cavitation number.

\section{Conclusion}

It is clear from the results in the article that a number of properties in the flow of different liquids (water vs. oil) through a transparent CD nozzle are similar and a number of properties are different. So

- the behaviour of the characteristic $\Delta p=\mathrm{f}(Q)$ in the region without cavitation is in accordance with Weissbach's theory of fluid mechanics. In the case of flow with cavitation, there is a break in the characteristic (typical entrainment of the characteristic in pumps),

- the beginning of cavitation is given by the same cavitation number for a given geometry of the nozzle and different liquids,

- the formation of cavitation is a function of the physical properties of liquids (density, dynamic or kinematic viscosity), which are a function of temperature, especially for oils (but the temperature is limited so that oil does not degrade),

- oil flow is laminar; water flow is turbulent,

- an interesting result is the values of the loss coefficient of the same element at different flowing media. The article can also serve as a methodology for evaluating losses on hydraulic components,

- the length of the cavitation cloud is observable in a transparent nozzle and depends on the flow value. At high flow rates, the dynamic behaviour of the cavitation cloud can be observed. Air cavitation occurs in the oil and in the initial phase it manifests itself only at a greater distance behind the narrowing of the nozzle,

- the cavitation cloud is more transparent and gas bubbles are more recognizable when oil cavitation is fully developed, but when cavitation on water, the cloud is dull, which is caused probably the small size of the bubbles,

Identification of cavitation and its influence on the frequency characteristics of noise and vibration, numerical simulations of multiphase flow of water in nozzle (the liquid mixture is 
given by the three phases, i.e. water, vapour, air) have been addressed in several articles [2, $3,7,8,9]$. The experiment will be further extended to measure noise and vibration as a method of identifying cavitation in opaque hydraulic elements (valves). Experimental results mainly for oil flow will be the basis for the specification of a mathematical model of oil flow with cavitation, where the multiphase fluid will be defined by only two components (oil, air) and will be used as boundary conditions for numerical calculations and to verify the created mathematical model.

\section{Acknowledgments}

This work was supported by the European Regional Development Fund in the Research Centre of Advanced Mechatronic Systems project, project number CZ.02.1.01/0.0/0.0/16_019/0000867 within the Operational Programme Research, Development and Education.

The work presented in this paper was supported by a grant SGS „,Research in the field of energysaving device for testing rotary hydraulic motors." SP2020/25.

\section{References}

1. J. Noskievič, Kavitace v hydraulických strojích. Praha, (1989)

2. L. Wen-Guang, Modeling Viscous Oil Cavitating Flow in a Centrifugal Pump. J. of Fluids Engineering 138(1) (2015) DOI: 10.1115/1.4031061

3. J. Jablonská, M. Kozubková and P. Marcalík, Experimental circuit for the generation of cavitation in oil flow. In Experimental Fluid Mechanics 2019. Františkokvy lázně 19.11.2019 - 22.11.2019

4. C. E. Brennen, Cavitation and bubble dynamics. Cambridge University Press, USA, (2014)

5. L. Pastrňák, Měreni hydraulických parametrů na olejové trati s dýzou. Bachelor thesis Ostrava, (2019)

6. P. Hodges Hydraulic fluid. ISBN 0340676523 New York, (2004)

7. J. Jablonská, and M. Bojko, Mathematical and experimental modelling of flow of airsaturated water through a convergent-divergent nozzle, EPJ Web of Conferences. 114, (2016) ISSN: 21016275. DOI: 10.1051/epjconf/201611402050

8. A. Bureček, L. Hružík, M. Vašina, Determination of undissolved air content in oil by means of a compression method. Strojniski Vestnik - J. Mechanical Engineering, 61, 78 (2015) doi: 10.5545/sv-jme.2015.247

9. R. Olšiak, M. Murgašová, M. Mlkvik, \& F. Ridzoň, The identification of cavitation in Kaplan turbine runner. AIP Conference Proceedings, volume 2118 , doi: $10.1063 / 1.5114762$ 